2. Hoeritzauer I, Pronin S, Carson A, Statham P, Demetriades AK, Stone J. The clinical features and outcome of scan-negative and scan-positive cases in suspected cauda equina syndrome: a retrospective study of 276 patients. J Neurol 2018;265:2916-26.

3. Leue C, Kruimel J, Vrijens D, Masclee A, Van Os J, Van Koeveringe G. Functional urological disorders: A sensitized defence response in the bladder-gut-brain axis. Nat. Rev. Urol. 2017;14:153-63.

\section{FEIGNED OR FUNCTIONAL?}

Mark Edwards. Eleanor Peel Chair for the Study of Ageing, Professor of Neurology and Honorary Consultant Neurologist, Atkinson Morley Regional Neuroscience Centre

10.1136/jnnp-2019-BNPA.12

Mark Edwards is Professor of Neurology at St George's University of London and Consultant Neurologist at the Atkinson Morley Regional Neuroscience Centre at St Georges University Hospital. He has a specialist clinical and research interest in Movement Disorders and in neurophysiological and psychophysical methods for exploring their pathophysiology. He did his $\mathrm{PhD}$ at the UCL Institute of Neurology with Professor John Rothwell and Professor Kailash Bhatia and was then a Senior Lecturer and Honorary Consultant Neurologist at UCL and the National Hospital for Neurology and Neurosurgery. Here he built up a specific interest in functional neurological symptoms and developed an NIHR funded research program and specialist clinical diagnostic and treatment service for patients with functional movement disorders. At St George's he is part of an integrated diagnostic and treatment service for functional neurological disorder, and continues also with research and clinical work in movement disorders in general.

Abstract 'Poor Hysterics...first they were treated as victims of sexual trouble...then of moral perversity and mediocrity...then of imagination'. Over a century since William James wrote these words, the status of people with functional neurological disorder remains uncertain and ambiguous. The language of everyday medical discourse betrays this ambiguity: 'Are they real seizures? ... Does he have genuine weakness?...The good news is that there's nothing serious wrong...' In this talk I will explore to what extent clinical and experimental work can help address this issue. While we may not be able to resolve the question of 'feigned or functional?' - perhaps because it is unanswerable in this form - exploring it may make us more aware of our own biases, hidden or not, and the way they affect our interaction and care for people with functional neurological disorder.

\section{HYPERMOBILITY AND AUTONOMIC DYSFUNCTION: INSIGHTS FROM BENCH TO BEDSIDE}

Jessica Eccles. Sussex Partnership NHS Foundation Trust and Brighton and Sussex University Hospitals NHS Trust

\subsection{6/jnnp-2019-BNPA.13}

Dr Jessica Eccles trained in medicine at University of Cambridge and University of Oxford, completing a BA in The History and Philosophy of Science, sparking a keen interest in philosophy of mind and brain-body interactions. Since graduation from medical school has pursued a combined academic clinical path at Brighton and Sussex Medical School. As an MRC Clinical Research Training Fellow she recently completed her $\mathrm{PhD}$ in the relationship between joint hypermobility, autonomic dysfunction and psychiatric symptoms and is now an NIHR Clinical Lecturer. She is currently working on an Academy of Medical Sciences grant to explore neural connectivity in hypermobility using leading edge Human Connectome Project techniques and has recently been awarded a MQ Arthritis Research UK Fellows Award to conduct a randomised clinical trial of a new targeted treatment for anxiety in hypermobility. Dr Eccles has also been awarded a grant from Dysautonomia International and will be working with Profs Critchley, Cercignani, Rowe, Murphy and Drs Nagai, Asslanni, Iodice and Giovanni to explore multi-modal correlates of 'brain fog' in Postural Tachycardia Syndrome. Dr Eccles is working with Profs Davies, Harrison, Cercignani, Critchley and Dr Tarzi to explore brain- body interactions in Fibromyalgia and ME/CFS. This involves autonomics, inflammatory and cytokine markers, brain imaging and genomics. This work is funded by Versus Arthritis and Action for ME Alongside clinical academic colleagues at BSMS, Prof Harrison and Dr Colasanti.

Joint hypermobility is a common, yet poorly recognised variant of connective tissue affecting up to $20 \%$ of the population. Hypermobility is a cardinal feature of Hyermobility Spectrum Disorder (HSD) and hypermobile Ehlers Danlos Syndrome (hEDS), inherited disorders of connective tissue. Individuals with joint hypermobility are over represented in panic, anxiety and neurodevelopmental populations and are prone to dysautonomia, typically postural tachycardia syndrome (PoTS), in which there is a phenomenological overlap with anxiety disorder. Interestingly differences in brain structure and function have been described in hypermobility in regions associated with emotional processing, including amygdala and insula. Individuals with joint hypermobility are more likely to experience severe chronic widespread pain, and many have co-morbid rheumatic conditions. A datadriven theoretical model linking joint hypermobility to psychiatric disorder is proposed, characterised by aberrant autonomic control and central representation, grounded in current theoretical models that seek to frame emotion as interoceptive inference, using leading-edge predictive coding approaches. Ultimately this approach has considerable relevance to personalised psychiatric medicine in this disorder and greater understanding of brain-body mechanisms underpinning neuropsychiatric states.

\section{THE LANGUAGE DISORDER IN SEMANTIC DEMENTIA: DOES IT MATTER WHICH LANGUAGE YOU SPEAK?}

Karalyn Patterson. University of Cambridge

\subsection{6/jnnp-2019-BNPA. 14}

From its inception, Karalyn Patterson has shaped the field of cognitive neuropsychology - the study of how the brain's structure and function relates to mental processes concerning the generation and use of knowledge. Specifically, Karalyn has used the effects of brain disease and injury to improve our understanding of language and memory. Her approaches are varied and rigorous, combining computer modelling and structural and functional brain imaging with observations of behaviour in normal and brain-damaged adults, as well as those affected by certain brain diseases. This has allowed Karalyn to directly link particular structures in the brain with specific 